\title{
THE GREEN GULLY REMAINS
}

\author{
By N. W. G. Macintosh \\ University of Sydney
}

\begin{abstract}
A summary is presented of Professor Macintosh's published accounts of his preliminary examination of the Green Gully remains. The physical condition of the bones, their excavation and laboratory treatment are described, together with some tentative assessment of their status and anomalous features.

\section{Introduction}

The archaeologists attempted a very thorough job of field recording of each stage of the excavation, by use of notes, diagrams, scale measurements of levels and surveys, still colour plus black and white photography, and colour motion picture film. Inclement weather, particularly rain, and a mishap to one camera resulted in close-up photographic studies of the bones in situ being somewhat short of perfection. Nevertheless, sufficient photographic record is available to indicate how the bones appeared to Casey and his team.
\end{abstract}

\section{Occurrence and Collection}

In these photographs there is no bone which does not show fracture, indeed multiple fractures, and secondly the anatomical relations of these bones one to another are much disturbed. For example, the clavicles are parallel instead of transversely in line; the right femur is dislocated out of the acetabulum and rotated through $180^{\circ}$; by diagrammatic projection of the residua of the shafts it seems obvious that the lower end of the right femur and the upper end of the right tibia must have been at least 16 in. apart; the dorsum of the right hand bones and the dorsum of the right forearm bones appear to be contiguous.

Such distortion of relationship simply does not happen following burial of an intact corpse in ground which is free from disturbance such as this appears to have been, i.e., an infilled grave marginally delineated from the surrounding medium of intact soil structure. Therefore it seems necessary to assume that it was not a corpse, but a skeleton which was buried some 12 months after death and after most of the flesh had gone from the bones. In other words, this would seem to have been a 'delayed burial'. Delayed burial is practised by aboriginal Australians today in Arnhem Land, and writers in the 1800 s recorded the custom in S. Victoria. The Green Gully bones would appear to indicate that the custom goes back some thousands of years. This is an astonishing example of the force of sanction, and persistence of traditional behaviour.

It can be seen also from the field photographs that the front-end loader sliced off the norma facialis of the skull, the inferior halves of the femora, superior halves of the tibiae and part of the shaft of the left humerus. These broken pieces rolled down the slope. Casey's men sieved the base of the slope and separately boxed all bone fragments and artifacts therefrom in 24 cardboard containers. (All human bone fragments from this collection down the slope have now been fitted to the fractured margins of bones taken from the grave).

The field workers were concerned about the removal and preservation of bones in the grave. "As the earth was removed from above them they were painted with a 
of bicipital groove and anterior aspects of the greater and lesser tuberosities. The problem at the lower end was more complicated; medial epicondylar area, lateral epicondylar area, and trochlea area were not only lying separate from themselves and from the shaft, but were also each found to be separated into an anterior and posterior Bedacryl-clogged mosaic of chips. Each portion was dealt with in accordance with the method described above. In joining the portions together, it was necessary to re-dissolve adhesive, and readjust some of the adjacent edges of chips already positioned, but now, free of the obscuring Bedacryl and matrix, this could be done with less risk of loss of the fragments or of failure to identify the margins of the chips; 37 fragments were successfully reassembled at the lower end, but a small ragged gap remains unrestored in the olecranon fossa and there is a small deficiency at the lateral epicondyle.

The three transverse fractures of the shaft were found to be welded together by very hard carbonate crust 1.0 to 4.0 (average $2 \cdot 0$ ) $\mathrm{mm}$ thick. It was obvious that cracks in the bone had been invaded by carbonate in ground water solution. The carbonate had deposited in the cracks and there aggraded, forcing the cracked fragments apart. This is quite certain, because plugs of this crust were found to extend up and down the marrow cavity for distances of 1.0 to $5.0 \mathrm{~cm}$ from the fracture lines. Hence these are ancient fractures and the question presented itself as to whether they were pre- or post-mortem breaks.

The crust was soaked with dilute acetic acid, but even so had to be drilled away with steel dental drills. The plugs in the marrow cavity were particularly recalcitrant. It was then found that the cleaned bone edges provided perfect alignment, and no artificial trimming of bone whatsoever was necessary for perfect fit. As right-angled notching in the lines of fracture occurred in five places, it appeared that these were post-mortem breaks. Plugs of trimmed balsa wood plus adhesive inserted into the marrow cavity completed reassemblage of the shaft, and this right humerus can be regarded as 95 per cent complete and hence is one of the very few bones in the total recovery that is available for measurements and subsequent calculations by formulae to assess the stature of the individual, and other deductions.

\section{Fractures}

Three types of fracture were recorded in the total bony remains. First, the ancient post-mortem fractures as described above, which were immediately identifiable by the accumulated grey crust on the fracture margins. Secondly, fissure fractures, particularly where the cortex was thin or where the bone had undergone decay and disintegration in the ground. Here there was no marginal crust accumulation, but the edges were dark brown. I have no reports so far on chemical, grain size or other analyses of the matrix, but the matrix when immersed in water and subsequently strained is seen to be fine grained and glutinous. On drying out it shrinks and cracks into plaques of a characteristic pattern. I would therefore think that expansile-contractile clay is involved and is probably the agent responsible for the multiple minute fractures and the multiple fragmentary bone chips. Thirdly, there were recent fractures (a) caused by the front-end loader, and these were severe, (b) others caused in field excavation, and these would seem to have been minimal, and (c) fractures due to squeezing by the applied protective wrappings as they contracted in drying out. This effect was severe on the ribs, which were mere cortical shells. All these recent fractures were obvious because of their fresh white margins. (A fourth type, pre-mortem, will be referred to later.)

The surfaces of the bones had only a trace of carbonate crust ranging from nil to $0.5 \mathrm{~mm}$, best seen on the inner table of the skull bones, adjacent to which the matrix showed minute beading of carbonate deposition. But here and there, for 
strengthening solution of Bedacryl in acetone and covered with several layers of tissue paper pasted on to protect them from the plaster of Paris used for reinforcement' (Bowler et al., 1967). Three blocks-the head, the trunk plus limbs, and the feet-were encased in a three-quarters or four-fifths surround of plaster of Paris; the three blocks were then undercut, and subsequently surrounded by foam rubber, and crated for transport. The theory in this preparation was that removal of plaster of Paris could be effected with ease or at least safety because of the underlying several layers of tissue paper, which in turn could be easily removed because clag is water-soluble; then the Bedacryl could be removed by dissolving with acetone.

Four factors operated against consummation of such intention. The plaster of Paris (surgical bandages) had been moulded to the shapes of the bones; in drying, the plaster bandages underwent shrinkage and tightly compressed the underlying layers of tissue paper to a single papier-mache-like layer also tightly moulded to the bone; the clag of the tissue paper, the Bedacryl and the clayey matrix had combined to form a compound which was not soluble in any of a number of reagents tried. Finally, this compound had, in some places, most firmly bound itself to the sub-periosteal basal lamina of the bone which scaled off under the least traction, thereafter defying identification. Much time was spent on experiments in the Department of Anatomy before we plunged into the actual operation, and indeed similar experimenting and modifications of technique continued right up to the moment of extraction of the last piece of bone from matrix, 2 January 1967.

\section{Cleaning and Conservation}

The problem was overcome by using a one-centimetre traverse oscillating saw, mounted in pistol grip and electrically driven, to fenestrate the plaster of Paris. The frames of these scores of small windows were then in turn cut through, and so pressure or wrenching effects on the underlying bones were avoided. It was ultimately found that 40 per cent xylol in acetone would convert the compound of clag-Bedacryl-matrix to a latex or chewing gum-like consistency, which could then be teased up with forceps and dissected off the bones; some dozens of HowardParker detachable blades were used up in this work. Details of this complex task of conservation and reassemblage of the various bones will be presented as a separate paper at some later date. Reference is here made only to the conservation and reconstruction of the right humerus. The field photographs permitted one to see three transverse fractures of the shaft more or less equi-distant, and the head of the humerus and the lower end appeared to be at least partially detached and fragmented.

After the coverings had been removed, it was found that a gap measuring $7 \cdot 0 \times$ $2.3 \mathrm{~cm}$ existed at the upper end of the shaft involving the region of the bicipital groove. Bone from this gap was adherent as a mosaic of chips to the inner aspect of the applied Bedacryl-clag-matrix. This area of wrappings was trimmed with scissors, laid on a broad spatula, supported around its margins so that the chips could not float away or otherwise disperse from one another, and the whole immersed in a lidded tray with a shallow $(1.0 \mathrm{~cm})$ depth of acetone and xylol. After 24 hours, each chip was separately removed in turn and joined progressively to its neighbouring chip. Had the chips become dispersed, it would not have been possible to identify all adjacent margins correctly; this gap in the shaft was completely restored by this reassemblage of a total of 13 fragments ranging in size from $0.3 \times 0.6 \mathrm{~cm}$ to $4.3 \times 1.3 \mathrm{~cm}$. One contact edge, $1 \mathrm{~cm}$ long, between shaft and head, was available at the postero-medial margin of the anatomical neck. Without this, repositioning of the head on the shaft would have been no better than an educated estimation. There remains a small deficiency of bone at the upper margins 
example on the right proximal phalanx of the great toe, at two places on the left femur, and elsewhere, there were isolated random plaques of hard crust 5 to $7 \mathrm{~mm}$. thick which had to be drilled off. This is instructive because it indicates the burial must have occurred at the very terminal part of that climatic period which was responsible for the carbonate-containing stratum, $6 \mathrm{ft}$ thick, in the top level of which the grave occurred.

\section{Bone Colour}

Progress photographs in black and white and also colour were taken at successive stages of removal of the bones from their protective wrappings and the contained matrix. Everything has been kept in separate containers, i.e. sediments, crust scrapings or drillings from individual bones, artifacts, gravel, adventitious rock pieces, and so on. The colour of the matrix was seen to be different in different regions of the skeleton. The upper trunk bones were enveloped in a light brownish to ochre coloured matrix with patches of pinkish-red and ash-dark-soil alternating in random fashion, minute charcoal chips and fine gravel intermingled. The lower trunk and upper femora rested in a more uniformly chocolate brown matrix and the feet in a very dark brown soil with considerable fine gravel. Soil filling the cranial cavity was distinctly and uniformly reddish brown.

The bones themselves show no sign whatsoever of firing; they are neither burnt nor smoke-stained. There is a pale bluish colouration on their surfaces (Tindale uses the term 'bluish bloom' to describe this appearance). This was not absolutely uniform, but its actual extent is now uncertain. It removes very readily by rubbing, scraping or polishing, exposing then a dirty white or light khaki surface. In removing the adherent Bedacryl-matrix-clag by dissection, particularly where it was intractably adfixed, this 'bloom' and also any carbonate film, could not be observed, but that is not to say it was not present. My impression at this post-operational stage is that the lower extremity bones exhibited pronounced and uniform 'bloom', the skull bones some patchy 'bloom', and the trunk and right upper extremity bones a trace.

\section{Bone Identification}

A normal skeleton has 236 bones counting individual skull bones, auditory ossicles, sesamoids, separate sacral, coccygeal and sternal segments. But the Green Gully bone fragments numbered 3,900 pieces. The task of identication has therefore been severe. Of these 3,900 pieces, only 730 could be identified and used for reassemblage. Some hundreds of other fragments were recognized partly from their appearance and partly from their relative positions in the matrix blocks, as being chips of ribs, thoracic vertebrae, and so on, but no reconstructive use could be made of them.

The 730 identified and usable fragments were immersed in 25 per cent Bedacryl in xylol, or in Tarzan's Grip q.s. in acetone, under vacuum for 24 to 48 hours to harden the bone. This technique was found, after many experiments, to give best results, and the product has sustained plastic and polyester resin replica production without harm.

These bones were then photographed separately and life-size prints were stuck down on a panel pari passu with the reassemblage of the fragments themselves by adhesive. From the actual reassemblage of the bones, it can be seen that these 730 pieces constitute approximately only two-fifths of a total skeleton, so it is neither 'almost complete' nor 'in good condition'.

All the remaining fragments were no larger than about a centimetre and have been utilized for specific tests. Two thousand seven hundred and eighty-two fragments, weighing $136 \mathrm{gm}$, were sent to Athol Rafter, Director of the Institute of 
Nuclear Sciences, D.S.I.R., New Zealand, for radiocarbon test. Eighty-six fragments, weighing $4.3 \mathrm{gm}$, from the feet bones and 16 pieces, weighing $5.0 \mathrm{gm}$, from thoracic vertebrae were sent to Dr K. P. Oakley, British Museum, for fluorinenitrogen-uranium test. Sixteen pieces, weighing 7.5 grams, from thoracic vertebrae and estimated to contain approximately $3.0 \mathrm{gm}$ of cancellous bone, were sent to Michael Charney, Colorado, for ABO group test together with separate samples of soil from the skull interior, the upper trunk and the feet.

Turning now to the 730 reassembled pieces constituting two-fifths of a skeleton, the following observations are necessarily tentative, because almost nine months of technical preparation of these bones, involving an average of 16 hours per week, has meant that one is only now in a position to begin an actual thorough study of them as a whole. At this point I pay tribute to my technical staff, Messrs. B. Bailey and S. L. Larnach (bone preparation and identification)), G. Williams (photography), K. Parsons (replicas and chemicals), P. Mills (drawings, panel, etc.), who in varying degrees shared the work with me.

Our first and somewhat alarming observation was that the left humerus is quite obviously on inspection and by measurement, much larger than the right humerus. Only the middle two-thirds of the shaft of the left humerus has survived, and it is broken near its mid-point. The inferior portion was recovered from the base of the slope where the front-end loader was working, while the superior portion came out of the grave matrix. The broken ends fitted together perfectly and the lower portion had obviously been dislodged by the front-end loader.

Actual measurements are confined to shaft diameters and in this the left is uniformly larger than the right humerus. The actual length of the surviving portion of the left shaft is greater than the length of the right between estimated analogous points. Approximately three-quarters of the left humeral head survives, and as far as present study has gone, it appears to have analogous dimensions, curvature and modelling with the right humeral head (which is intact). The glenoid fossae of the right and left scapulae (which are fragmentary) have equal dimensions. The right and left clavicles are approximately equal in dimensions, slender, rather small, and suggest female sex. It seems necessary to conclude that the right humerus and the left humeral shaft derive from two individuals, not from one.

The bones of the lower extremities appear to be disproportionately large and rugged when compared with the right upper extremity bones, with the clavicles, with the remnants of the scapulae, with the rib fragments, and with the skull.

The pelvic bones have not survived, but small fragments of external plate of ilium were recognized, and then, part of a femoral head and a small portion of acetabular fossa were identified. Our present opinion is that the latter two pieces are mutually adaptable in curvature, size and fit. More study is necessary to confirm or deny this very important question. Subsequently Casey has loaned me a small series of Kodachrome frames which I had not seen previously. They show the bones in situ in the field, and the colour definition is good. The pelvic bones were among those referred to by the field workers as mere ghosts in the matrix, but they appear in these frames to be more like the bones of the lower extremities in having a pronounced blue 'bloom', whereas the thoracic and right upper extremity bones show none, and the skull some mottled blue.

\section{Pathology}

The present stage of study indicates that the remains show no evidence of pathology except for the following:

1. Pre-mortem fractures are present in the right ulna $7.0 \mathrm{~cm}$ above its distal end and in the right radius some $5.0 \mathrm{~cm}$ above its distal end. These fractures had 
been severe, probably comminuted and possibly compound with anterior and lateral displacement of the inferior portions. Union (with or without treatment) had occurred in bad position with overlap, and thus shortening, of 1.0 to $1.5 \mathrm{~cm}$, and each fracture site had healed with marked sigmoid curvature, particularly the ulna fracture. This probably involved some limitation of right wrist function and so might possibly call for further thinking about the more massive left humeral shaft.

2. A foramen is present in the middle of the articular surface of the distal end of the left tibia. The lower end of this left tibia is represented by a fragment only some $4.0 \mathrm{~cm}$ in length. Analogous bone from the right side has not survived, so it is unknown whether or not the feature was bilateral. The foramen leads into a canal which traverses as much of the fragment as has survived. I can find no reference to such a foramen in the literature. Photographic magnifications of the aperture suggest bone reaction at the margins, but might equally represent digestion and secretion or excretion effects of some worm-like creature. It has been examined by Davies of London, Sunderland and Ray of Melbourne, and pathologists from Melbourne, Brisbane and Sydney. We cannot interpret it.

3. Surviving teeth are excessively worn occlusally, even for an aboriginal, so either the diet was very harsh or she used her teeth excessively in chewing fibre to make nets, string, etc. In an aboriginal female this degree of wear would suggest an age of 40-45. But the sutures of her skull suggest an age of only 30-35. These teeth are small, falling inside recorded size range of aboriginal female teeth and outside the size range of male aboriginal teeth (Macintosh and Barker 1965). Three complete teeth and fragments of roots and crowns of perhaps four or even five more have been recovered. Of these the right upper lateral incisor and canine and two root apices of first premolar are in situ in portion of the right maxilla. A plastic cast of the matrix mould which had partially surrounded the missing right upper incisor enables its dimensions to be calculated.

\section{Interpretation}

This is not the place, nor has sufficient study been completed, to deal with all the anatomical detail, measurements, formulae and calculations. Instead, I say simply for the moment that the left humeral shaft and the bones of the two lower extremities (and maybe the pelvis) in appearance and measurements suggest male, and all the rest is female. The skull is female; there is no question about that.

Compared with aboriginal measurements and statistics from N.S.W. skeletons and from data in the literature, the Green Gully presumptive male bones would seem to fall high within male ranges. The presumptive female Green Gully bones would seem to fall low within male ranges and high within female ranges. We need now to compare the Green Gully skull with other Australian prehistoric crania. Of these there are only four which are significant, viz., the Talgai cranium from Queensland, the Cohuna cranium from the Murray River region, the Mossgiel skull from far western N.S.W., and the Keilor cranium from only two miles away. They are each male, which makes the comparison more difficult.

The Green Gully skull, although gracile, is quite large for a female but significantly smaller than Keilor, Cohuna and Mossgiel. In shape it is not at all like Talgai, Mossgiel or Cohuna, but it is very like the Keilor cranium. When photographically enlarged to the same size as the Keilor cranium, and then superimposed on Keilor photographs, the similarity is striking. Some discrepancy exists at the occipital region which may be a reflection of sex difference, or may turn out to be inaccuracy in our reconstruction. This was the last region assembled, and it was hurried. We propose to dissolve the adhesive and re-study the alignment of the edges of the fragments. Assuming for the moment that later study will confirm this 
identification of a male and female assemblage of bones, it is necessary to note that only the right humerus, the bones of the right second manual digit and the proximal phalanx of the right great toe are length-wise intact and these constitute a meagre basis for stature calculations by formulae. Extrapolations from circumferences and horizontal diameters give some assistance. From these scanty data, the tentative suggestion is made that the male may have been about $5 \mathrm{ft} 7$ in., which is a little above aboriginal average, and the female may have been about $5 \mathrm{ft} 5 \frac{1}{2}$ in., which is tallish for an aboriginal woman.

Once again, assuming that more meticulous study confirms these tentative decisions, how is this astonishing and unique find to be explained? First, no bone in the assemblage is duplicated. Secondly, the assessed male bones form a block consisting of lower extremities, probably pelvis, and left upper extremity. The assessed female bones form a unit of head and neck, thorax, right upper extremity, and both shoulder girdles. There is absolutely no evidence of departure from these two units, no evidence of a random melange of non-matching individual bones. Thirdly, the vertebral column is represented by the first and second and perhaps a piece of third cervical vertebrae; by a massive collection of thoracic vertebral fragments, identified almost solely on their position in the matrix; by a maximum of three fragments of lumbar vertebrae derived from (?) the third and fifth, and perhaps a fragment of sacral promontory. In other words there is a gap in vertebral representation between upper cervical and upper thoracic, and also between lower thoracic and mid or lower lumbar. Those are the observed facts in an admittedly most fragmented and incomplete assemblage of bones. Do these observed facts nevertheless lend themselves to interpretation?

Only one explanation will satisfy the circumstances: A male and a female body were adjacently exposed while awaiting their 'delayed burials'. When nature had reduced the bodies to bones, the aboriginal mourners gathered up and buried in one grave some two-thirds of the female and about one-third of the male. The obvious question arises, was this by accident or design? We have no means of deciding with certainty, but I favour the view that it was accidental. The Sydney police have brought to me from time to time remains of individuals who had died of exposure on the western plains, or nearer to Sydney in the Hawkesbury scrub. Remnants of skin and tendons and ligaments, although fenestrated by carrion beetle, had become semi-tanned or semi-mummified by the elements and served to hold together a majority of the bones of the limb concerned. Two such tattered arms mounted in perspex by my Department can be seen in the Scientific Branch of the Sydney C.I.D. The remainder of the individual was not found, apparently having been dispersed by crows, hawks, or eagles. The proximal bones ended in oblique fracture lines, not unlike that at the proximal end of the Green Gully left humeral shaft. I visualize the remains of the male and female corpses awaiting 'delayed burial' being similarly disturbed by predators. When the mourners came to inter one of the individuals, they were faced with the task of selecting and arranging the remains of one individual from the partly separated and scattered remains of two individuals, and not being anatomists they got the limbs mixed.

It is easy to visualize the head detaching itself from the trunk at the cervical region; indeed one would expect this, also to visualize the body separating at the lumbar region into an upper portion and a lower portion. The bones of these separated portions might well be maintained in loose relationship to one another by air-mummified tendons and ligaments. These visualizations may sound contrived, but their occurrence is relatively commonplace in forensic experience. In any case, what other explanation will fit all the observations? It is of course possible to argue deliberate intent, stemming from any emotion ranging from love to punitive revenge. In conclusion, it can only be repeated that much more anatomical study is 
necessary, before arriving at definitive opinion. Study so far has been almost totally limited to observations incidental to the enormous task of extracting the bones from their matrix and field coverings and to the conservation treatment, identification and assembly of the 730 usable fragments which constitute a grievously incomplete representation of only two-fifths of a total skeleton which in turn presents considerable evidence that it derives from the upper part of a female and the lower part of a male, that is, two different individuals.

Meanwhile, I feel it is safe to say that the assumed male and female bones, while large, are within the ranges recorded for modern aboriginals. The skull is Keilor-like, not Talgai-Cohuna-Mossgiel-like. The Keilor cranium undoubtedly is somewhat older, but whatever its age its morphological configuration has in general terms persisted until 6,460 years ago in S. Victoria, as exhibited by the Green Gully remains.

\section{References}

Bowler, J. M., Mulvaney, D. J., Casey, D. A., and Darragh, T. A. 1967. Nature 213: 152. Macintosh, N. W. G., and BARKer, B. C. W., 1965. The Osteology of Aboriginal Man in Tasmania. Oceania Monograph, 12.

\section{Note}

This account of the Green Gully remains has been edited with the author's consent from his Presidential Address, Section F, ANZAAS Congress, Melbourne 1967. (Aust. J. Sci. 30: 86-98). In a later address to the Australian College of Dental Surgeons, Canberra, 1967, Professor Macintosh provided the additional information that tests performed by Michael Charney, University of Colorado, U.S.A., indicated 'that a small sample of bone and of matrix each gave testresults for blood Type O'. (Ann. Aust. Coll. Dent. Surg. 1: 108-126). 


\section{$2 \mathrm{BHL}$ Biodiversity Heritage Library}

Macintosh, N W G. 1970. "The Green Gully remains." Memoirs of the National Museum of Victoria 30, 93-100. https://doi.org/10.24199/j.mmv.1970.30.06.

View This Item Online: https://www.biodiversitylibrary.org/item/120624

DOI: https://doi.org/10.24199/j.mmv.1970.30.06

Permalink: https://www.biodiversitylibrary.org/partpdf/258273

\section{Holding Institution}

Museums Victoria

\section{Sponsored by}

Atlas of Living Australia

\section{Copyright \& Reuse}

Copyright Status: Permissions to digitize granted by rights holder.

This document was created from content at the Biodiversity Heritage Library, the world's largest open access digital library for biodiversity literature and archives. Visit BHL at https://www.biodiversitylibrary.org. 